\title{
PROFILAXIA DAS ÚLCERAS ASSOCIADAS AO ESTRESSE
}

\author{
Prophylaxis of ulcers associated with stress \\ Carlos Eduardo POMPILIO, Ivan CECCONELLO
}

Trabalho realizado no Departamento de Gastroenterologia do Hospital das Clínicas da Faculdade de Medicina da Universidade de São Paulo - USP, São Paulo, SP, Brasil

DESCRTORES - Úlcera duodenal. Terapêutica. Inibidores da bomba de prótons.
RESUMO - Introdução - As úlceras da mucosa gastroduodenal que aparecem após graves insultos têm sido chamadas de úlceras associadas ao estresse ou, mais genericamente, de "doença mucosa associada ao estresse" (DMAE). São conhecidas desde a Antiguidade e apesar de relacionarem-se com altos índices de morbidade e mortalidade, esquemas de profilaxia no intuito de minimizar seus riscos, só foram introduzidos recentemente. Os inibidores da bomba de prótons têm desempenhado papel importante nesse cenário. Métodos - O presente artigo é uma breve revisão sobre seu uso dentro do contexto clínico da profilaxia do sangramento gastrointestinal relacionado ao estresse.

\section{Correspondência: \\ Ivan Cecconello \\ e-mail: icecconello@hotmail.com}

Fonte de financiamento: não há

Conflito de interesses: não há

Recebido para publicação: 16/11/2009

Aceito para publicação: 15/12/2009

HEADINGS - Duodenal ulcer. Therapeutics. Proton pump inhibitors.
ABSTRACT - Introduction - Gastroduodenal mucosal ulcers that appear after severe insults have been called ulcers associated with stress or, more generally, "mucosal disease associated with stress" (DMAE). Are known since antiquity and although linked with high morbidity and mortality, prevention schemes in order to minimize their risk, were introduced only recently. The proton pump inhibitors have played an important role in this scenario. Methods - This article is a brief review of its use within the clinical context of prophylaxis of gastrointestinal bleeding due to stress.

\section{INTRODUÇÃO}

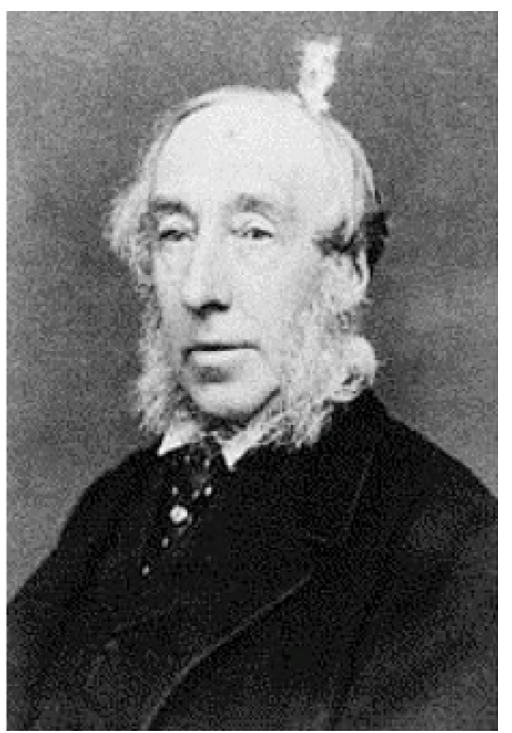

Thomas Blizard Curling (1811-1888)
A s úlceras da mucosa gastroduodenal que aparecem após graves insultos - como queimaduras, choque séptico e grandes traumatismos, por exemplo -, têm sido chamadas de úlceras associadas ao estresse ou, mais genericamente, de doença mucosa associada ao estresse (DMAE). São conhecidas desde a Antiguidade e apesar de relacionarem-se com altos índices de morbidade e mortalidade, esquemas de profilaxia no intuito de minimizar seus riscos, só foram introduzidos recentemente. Os inibidores da bomba de prótons (IBP) têm desempenhado um papel importante nesse cenário. O presente artigo é uma breve revisão sobre seu uso dentro do contexto clínico da profilaxia do sangramento gastrointestinal (GI) relacionado ao estresse.

\section{Curling, Cushing e Além}

A primeira descrição de DMAE foi de Curling em $1842^{7}$. Esse autor descreveu úlceras duodenais associadas a queimaduras como uma entidade clínica à parte do espectro clássico da doença péptica, embora ele mesmo admita que Dupuytren, dez anos antes, tenha chamado a atenção para ulcerações intestinais em pacientes que sobreviviam de lesões produzidas por queimaduras. Quase um século se passou para que um novo dado pudesse ser acrescentado a essa questão. Segundo Harvey Cushing, foi Carl von Rokitansky quem primeiro chamou a atenção para a associação entre ulceração péptica e lesões cerebrais ou meníngeas. Em seu estudo clássico de $1932^{\circ}$, Cushing mostrou onze casos de patologia cerebral nos quais, à autopsia, foram encontradas ulcerações no trato 
gastroduodenal superior. Por essa razão, o tipo de lesão gastroduodenal que associa hipertensão intracraniana e complicações graves do sistema nervoso central é conhecido como úlcera de Rokitansky-Cushing.

A DMAE foi descrita em 1971 por Lucas $^{12}$ que utilizou o termo stress-related erosive syndrome. Desde então, vários nomes têm sido utilizados para descrever a lesão da mucosa gastroduodenal pelo estresse, a saber, úlceras de estresse, erosões por estresse, gastrite por estresse, gastrite hemorrágica, gastrite erosiva e stressrelated mucosal disease, o termo pelo qual o espectro clínico é conhecido em língua inglesa ${ }^{3}$. Hoje, sabemos que são muitas as situações clínicas relacionadas a lesões por estresse da mucosa do trato gastroduodenal superior (Tabela 1). A fisiopatologia dessas lesões em situações de estresse clínico, já teve vários de seus passos principais elucidados, sendo que os pontos em comum permitem que as várias causas sejam agrupadas sob o termo DMAE.

TABELA 1 - Causas de úlceras relacionadas ao estresse

\begin{tabular}{|l|c|}
\hline Causas de úlceras relacionadas ao estresse & Odds ratio \\
\hline Insuficiência respiratória & 15,6 \\
\hline Coagulopatia & 4,3 \\
\hline Sepse & 2,0 \\
\hline Hipotensão & 3,7 \\
\hline Insuficiência hepática & 6,67 \\
\hline Insuficiência renal & 3,03 \\
\hline Glicocorticóides & - \\
\hline Uso prolongado de sonda nasogástrica & 2,59 \\
\hline
\end{tabular}

A isquemia da mucosa gastroduodenal parece mesmo ter um papel preponderante na DMAE, embora a progressão da lesão requeira meio ácido para ocorrer. Várias das causas descritas acima têm como mecanismo de lesão comum a redução do fluxo sanguíneo dirigido à mucosa gástrica ${ }^{11}$. Sabe-se também, que a reperfusão da mucosa após o evento isquêmico, aumenta a lesão tecidual em comparação com a isquemia sozinha, mecanismo mediado por radicais livres do oxigênio.

Há modelos experimentais bem estabelecidos de choque e ressuscitação que provocam lesões gástricas em ratos $^{18}$. Nesses modelos, é praticamente impossível provocar lesões histológicas grosseiras na mucosa sem instilar ácido. Concentrações maiores, provocam lesões mais graves sugerindo um mecanismo de doseresposta. Há indícios de que os sais biliares podem potencializar a agressão ao epitélio gástrico. À quebra da integridade da mucosa gastroduodenal ocasionada pela isquemia associam-se os distúrbios da motilidade gástrica e as alterações da secreção ácida causada por medicamentos ou mesmo pelo jejum, constituindo as causas fisiopatológicas mais importantes no estabelecimento das lesões de estresse.

\section{População de Risco}

A população de pacientes de terapia intensiva se constitui, pelo exposto, em um grupo vulnerável à DMAE. A incidência nesses pacientes varia bastante já que vários critérios são utilizados para definir a doença. Hoje, prefere-se o conceito de sangramento clinicamente relevante conforme definido por Cook como sendo um sangramento evidente seguido nas próximas 24 do evento pelo menos por um dos itens abaixo: (1) queda espontânea da pressão sistólica de $20 \mathrm{~mm} \mathrm{Hg}$ ou mais; (2) aumento de 20 batimentos ou mais na na frequência cardíaca; ou (3) hipotensão postural; ou (4) queda na hemoglobina de $2 \mathrm{~g} / \mathrm{dL}$ ou mais com necessidade de transfusão, sem um aumento subsequente compatível com o número de unidades transfundidas. A tabela 2 mostra as situações clínicas mais comuns nas quais a DMAE pode estar presente. A incidência de sangramento clinicamente relevante nessa população de risco varia de 3 a $4 \%$, com mortalidade atribuída de quase $50 \%{ }^{5}$, se constituindo no racional para a instituição da profilaxia.

TABELA 2 -População de risco para úlceras associadas ao estresse em terapia intensiva

População de risco para úlceras associadas ao estresse

Ventilação mecânica por mais de $48 \mathrm{~h}$

Coagulopatia (plaquetas $<50.000$, TTPA $>2 x$ ou INR $>1,5$

Instabilidade hemodinâmica

Queimaduras graves

Traumatismo crânio-encefálico

\section{Profilaxia}

A profilaxia da DMAE pode ser feita ao se atacar os três itens principais da fisiopatologia básica da doença, a saber: a produção de ácido, a estase gástrica e a correção dos distúrbios hemodinâmicos que levam à quebra da integridade da mucosa gastroduodenal. $\mathrm{O}$ estabelecimento da realimentação precoce, o uso de procinéticos e toda a ciência envolvida na ressuscitação hemodinâmica de pacientes em choque estão fora do escopo desse artigo. Focaremos nossa atenção à inibição da produção de ácido pela mucosa gástrica.

A produção de ácido pela mucosa gástrica e o conseqüente aumento do $\mathrm{pH}$ intraluminal pode ser efetivamente conseguido através do uso de duas classes principais de medicamentos: os antagonistas do receptor da histamina $\mathrm{H} 2(\mathrm{ARH} 2)$ e os inibidores da bomba de prótons (IBP), não por acaso, as principais drogas utilizadas para profilaxia da DMAE.

ARH2 (antagonistas do receptor da histamina $\mathrm{H} 2$ )

Os ARH2 utilizados para profilaxia da DMAE no Brasil são a cimetidina (a menos potente), a ranitidine (intermediário) e a famotidina (a mais potente). Nos EUA, a cimetidina é a única aprovada pela US Food and Drug Administration (FDA) para prevenção de sangramentos gastrointestinais. A cimetidina parece ser a que está mais associada a interações medicamentosas, talvez por ser a mais antiga e por ter sido mais estudada. Entre os efeitos colaterais descritos encontramos plaquetopenia, alterações neurológicas em idosos e alterações significativas das funções hepatica e renal. A 
ranitidina é 5 a 12 vezes mais potente que a cimetidina e a famotidina é 8 a 10 vezes mais potente que a ranitidina. A famotidina parece ter menos interações medicamentosas ${ }^{1}$.

As infusões contínuas de $\mathrm{ARH} 2$ são mais eficazes em manter o $\mathrm{pH}$ intraluminal gástrico acima de 4 que as intermitentes. Por outro lado, as preparações VO parecem ser seguras em manter o nível sérico das drogas quando comparadas com as preparações parenterais, ambas administradas de forma intermitente.

$\mathrm{O}$ grande problema dos $\mathrm{ARH} 2$ é a tolerância que se desenvolve em média $42 \mathrm{~h}$ após a administração venosa ${ }^{13}$. Uma revisão da American Health Pharmacist sobre a eficácia dos $A R H 2$ na prevenção da DMAE, realizada 10 anos atrás, não chegou a um consenso sobre seu uso 2 .

\section{IBP (inibidores da bomba de prótons)}

Os IBP são os mais potentes agentes disponíveis para inibição da secreção ácida do estômago. O omeprazolfoio primeiroIBP a serlançado evários estudos confirmaram sua eficácia em prevenir sangramento GI. O esomeprazol é um isômero do omeprazol e parece ter menos interações medicamentosas que esse ${ }^{1}$. Sua administração intravenosa é eficaz na profilaxia da DMAE em pacientes com insuficiência respiratória submetidos à ventilação mecânica.

Dentre os IBP, o pantoprazol tem o pH de ativação mais baixo e a maior estabilidade. Esses fatos e sua potência e disponibilidade de preparações parenterais, fazem dele uma importante arma no controle da $D_{M A E}{ }^{16}$. Um estudo recente ${ }^{14}$ envolvento 200 pacientes de terapia intensiva estudou o pantoprazol IV (40 mg uma vez ao dia, $40 \mathrm{mg} \mathrm{12/12} \mathrm{h,} 80 \mathrm{mg}$ uma vez ao dia, $80 \mathrm{mg} \mathrm{12/12} \mathrm{h} \mathrm{e} 80 \mathrm{mg} 8 / 8 \mathrm{~h}$ ) versus cimetidina em infusão contínua ( $300 \mathrm{mg}$ bolus; $50 \mathrm{mg} / \mathrm{h}$ ) em pacientes sob risco para DMAE. O end point primário foi o tempo durante o qual o pH gástrico era mantido acima de 4 . Os grupos de pantoprazol de $80 \mathrm{mg} \mathrm{12/12} \mathrm{h} \mathrm{e} 80 \mathrm{mg}$ $8 / 8 \mathrm{~h}$ mostraram os melhores efeitos, sem relação com o dia de tratamento. No dia 2, os grupos de tratamento

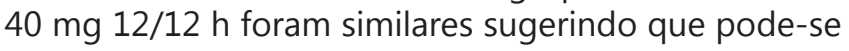
obter um controle adequado com dosagens mais altas no início seguidas de ajustes para dosagens mais baixas a partir de $48 \mathrm{~h}$. Todos os 5 grupos do pantoprazol foram mais eficazes que o grupo da cimetidina em infusão continua ${ }^{14}$.

Têm-se demonstrado que um $\mathrm{pH}$ maior que 4 é suficiente para previnir sangramento GI associado ao estresse. Entretanto, alguns estudos mostraram que pHs maiores que 6 são necessários para manter o coágulo em úlceras sangrantes evitando assim, o ressangramento. Essa habilidade dos IBPs em conseguir pHs gástricos maiores deve ser levada em consideração quando o risco de ressangramento for alto ${ }^{8}$.

A Tabela 3 mostra uma comparação entre os IBPs e os ARH2s. O uso de IBPs para profilaxia de sangramento GI relacionado ao estresse em pacientes de terapia intensive têm sido criticado em geral pela disseminação de sua prática e em especial pelo prolongamento de sua prescrição após encerrada a fase de maior risco $0^{6,10,15,17}$. Pelo exposto, o uso judicioso dessa poderosa ferramenta em pacientes selecionados parece estar indicado. São necessários novos estudos para podermos definir o papel dos IBPs principalmente nos pacientes de alto risco.

TABELA 3 - Comparação entre ARH2s e IBPs para profilaxia de úlceras de estresse

\begin{tabular}{lll}
\hline & Bloqueadores $\mathrm{H} 2$ & $\begin{array}{l}\text { Inibidores de } \\
\text { bomba } \mathrm{H}+\end{array}$ \\
\hline $\begin{array}{l}\text { Potência em reduzir a secreção } \\
\text { de ácido gástrica }\end{array}$ & ++ & ++++ \\
\hline Efeitos colaterais comuns & +++ & ++ \\
\hline Risco de pneumonia & ++ & ++++ \\
Interações medicamentosas & +++ & + \\
\hline Tolerância & + & - \\
Custo-efetividade & ++++ & ++ \\
\hline Efetividade terapêutica & ++ & ++++ \\
\hline
\end{tabular}

Modificado de Ali, T. (2009)1

\section{REFERÊNCIAS}

1. Ali T, Harty RF. Gastroenterol Clin N Am 38 (2009) 245-265

2. ASHP therapeutic guidelines on stress ulcer prophylaxis. ASHP commission on therapeutics and approved by the ASHP board of directors on 1998. Am J Health Syst Pharm 1999;56:347-79.

3. Choung RS, Talley NJ. Epidemiology and clinical presentation of stress-related peptic damage and chronic peptic ulcer. Curr Mol Med 2008;8:253-7.

4. Cook DJ, Fuller HD, Guyatt GH, et al. Risk factors for gastrointestinal bleeding in critically ill patients. Canadian Critical Care Trials Group. N Engl J Med 1994; 330:377-81.

5. Cook DJ, Griffith LE, Walter SD, et al. The attributable mortality and length of intensive care unit stay of clinically important gastrointestinal bleeding in critically ill patients. Crit Care 2001;5:368-75.

6. Craig DG, Thimappa $R$, Anand V, Sebastian S. Inappropriate utilization of intravenous proton pump inhibitors in hospital practice--a prospective study of the extent of the problem and predictive factors. QJM. 2010 Mar 7. [Epub ahead of print]

7. Curling TB. On acute ulceration of the duodenum in cases of burn. Med Chir Trans (Lond) 1842;25:260-81.

8. Fennerty MB. Pathophysiology of the upper gastrointestinal tract in the critically ill patient: rationale for the therapeutic benefits of acid suppression. Crit Care Med 2002;30:S351-5.

9. H. Cushing. Peptic ulcer and the interbrain. Surg Obst, 1932, 55: 1-34.

10. Jacob G. Hoover $Æ$ Annabel L. Schumaker, Kevin J. Franklin Use of Intravenous Proton-Pump Inhibitors in a Teaching Hospital Practice. Dig Dis Sci (2009) 54:1947-1952.

11. Laine L, Takeuchi K, Tarnawski A. Gastric mucosal defense and cytoprotection: bench to bedside. Gastroenterology 2008;135:42.

12. Lucas CE, Sugawa C, Riddle J, et al. Natural history and surgical dilemma of "stress" gastric bleeding. Arch Surg 1971;102:266-73.

13. Mathot RA, Geus WP. Pharmacodynamic modeling of the acid inhibitory effect of ranitidine in patients in an intensive care unit during prolonged dosing: characterization of tolerance. Clin Pharmacol Ther 1999;66:140-51.

14. Somberg L, Morris J Jr, Fantus R, et al. Intermittent intravenous pantoprazole and continuous cimetidine infusion: effect on gastric $\mathrm{pH}$ control in critically ill patients at risk of developing stress-related mucosal disease. J Trauma 2008;64:1202-10. 
15. Soumana C Nasser, Jeanette G Nassif, Hani I Dimassi. Clinical and cost impact of intravenous proton pump inhibitor use in non-ICU patients. World J Gastroenterol 2010 February 28; 16(8): 982-986.

16. Spirt MJ. Stress-related mucosal disease: risk factors and prophylactic therapy. Clin Ther 2004;26:197-213.

17. Thomas L, Culley EJ, Gladowski P, Goff V, Fong J, Marche SM. Longitudinal analysis of the costs associated with inpatient initiation and subsequent outpatient continuation of proton pump inhibitor therapy for stress ulcer prophylaxis in a large managed care organization. J Manag Care Pharm. 2010 Mar;16(2):122-9.
18. Yasue N, Guth PH. Role of exogenous acid and retransfusion in hemorrhagic shock-induced gastric lesions in the rat. Gastroenterology 1988;94(Suppl 1):1135-1143. 